\title{
Neurophysiological Correlates of Emotion Regulation in Children and Adolescents
}

\author{
Marc D. Lewis ${ }^{1}$, Connie Lamm¹, Sidney J. Segalowitz ${ }^{2}$, Jim Stieben ${ }^{3}$, \\ and Philip David Zelazo ${ }^{1}$
}

\begin{abstract}
Psychologists consider emotion regulation a critical developmental acquisition. Yet, there has been very little research on the neural underpinnings of emotion regulation across childhood and adolescence. We selected two ERP components associated with inhibitory control-the frontal N2 and frontal P3. We recorded these components before, during, and after a negative emotion induction, and compared their amplitude, latency, and source localization over age. Fifty-eight children 5-16 years of age engaged in a simple go/no-go procedure in which points for successful performance earned a valued prize. The temporary loss of all points triggered negative emotions, as confirmed by self-report scales. Both the frontal N2 and frontal P3 decreased in amplitude and latency with age, consistent with the hypothesis of increasing cortical efficiency. Amplitudes were also greater following
\end{abstract}

\section{INTRODUCTION}

One of the most important tasks of childhood is learning to regulate the impulses that accompany negative emotions. This capacity is referred to as emotion regulation, a suite of controls that permit adaptive functioning in the presence of emotions such as anxiety, anger, shame, distress, and even intense excitement. Under the rubric of emotion regulation, developmental psychologists have studied normative advances in children's abilities to inhibit emotional impulses, modulate emotional behavior, maintain engagement with important features of the world, and disengage from features that are emotionally distressing (Posner \& Rothbart, 1998; Derryberry \& Rothbart, 1997; Thompson, 1994). Moreover, individual differences in emotion regulation have been found to predict social competence and personality development (e.g., Kochanska, Murray, \& Harlan, 2000). Despite the importance of emotion regulation for normal development, very little is known about its neural underpinnings over the first two decades of life. Therefore, we looked at cortical activities mediating response inhibition-a key aspect of emotion

\footnotetext{
${ }^{1}$ University of Toronto, ${ }^{2}$ Brock University, ${ }^{3}$ York University
}

the emotion induction, only for adolescents for the N2 but across the age span for the frontal P3, suggesting different but overlapping profiles of emotion-related control mechanisms. No-go N2 amplitudes were greater than go N2 amplitudes following the emotion induction at all ages, suggesting a consistent effect of negative emotion on mechanisms of response inhibition. No-go P3 amplitudes were also greater than go P3 amplitudes and they decreased with age, whereas go P3 amplitudes remained low. Finally, source modeling indicated a developmental decline in central-posterior midline activity paralleled by increasing activity in frontal midline regions suggestive of the anterior cingulate cortex. Negative emotion induction corresponded with an additional right ventral prefrontal or temporal generator beginning in middle childhood.

regulation-during a task that elicited negative emotions. Specifically, we measured event-related potentials (ERPs) associated with inhibitory control across a broad age range and tested for differences in their amplitude, latency, and source localization with age and emotion condition.

Although there has been almost no research examining the neurocognitive correlates of children's emotion regulation, adults are thought to regulate their emotions through attentional controls mediated by the prefrontal cortex (PFC) and related paralimbic structures such as the anterior cingulate cortex (ACC) (Bishop, Duncan, Brett, \& Lawrence, 2004; Ochsner et al., 2004; Lange et al., 2003; Wong \& Root, 2003; Beauregard, Levesque, \& Bourgouin, 2001; O’Doherty, Kringelbach, Rolls, Hornak, \& Andrews, 2001; Simpson, Drevets, Snyder, Gusnard, \& Raichle, 2001; Davidson \& Irwin, 1999; Bechara, Damasio, Tranel, \& Damasio, 1997). Moreover, neuroscientists have begun to study developmental differences in these attentional controls, with particular emphasis on response inhibition. Despite some evidence for posterior cortical involvement for younger children (Bunge, Dudukovic, Thomason, Vaidya, \& Gabrieli, 2002), attentional control is generally associated with prefrontal activities at all ages. Moreover, 
several studies indicate less prefrontal activation as children mature. Neuroimaging research shows less prefrontal activation in adults than in children (Durston et al., 2002; Casey et al., 1997) and adolescents (Luna et al., 2000) in tasks requiring inhibition or directed attention. This reduction may be explained by increasing neural efficiency, such that less neural processing is necessary to achieve successful performance later in development (Casey, Giedd, \& Thomas, 2000). It may also indicate that synaptic pruning and myelination underlie increasing cognitive integration over development (Casey et al., 2000; Luna et al., 2000). ERP findings are partly consistent with this picture in that a number of studies point to decreased frontal ERP amplitudes with age. However, before reviewing these findings, it is necessary to (1) specify which ERP components are associated with frontally mediated mechanisms of response inhibition and (2) determine how they might be related to emotion.

Only two ERP components have been consistently linked with response inhibition. The first is a frontal N2, observed about 200-400 msec poststimulus at medialfrontal sites. The frontal N2 is generally reported on successful no-go trials (Falkenstein, Hoormann, \& Hohnsbein, 1999; Eimer, 1993; Jodo \& Kayama, 1992) and is thus referred to as the "inhibitory" or no-go N2. However, robust N2s have been found on go trials as well (e.g., Davis, Bruce, Snyder, \& Nelson, 2003; Nieuwenhuis, Yeung, Van den Wildenberg, \& Ridderinkhof, 2003). Although the frontal N2 is usually interpreted in terms of response inhibition or anticipatory attention, some authors propose that it marks the monitoring of conflict between competing responses or task representations (Nieuwenhuis et al., 2003; Botvinick, Braver, Barch, Carter, \& Cohen, 2001). Thus, the N2 might be considered an "evaluative negativity," whose psychological functions include effortful attention or action monitoring (Tucker, Luu, Desmond, et al., 2003). The second ERP component associated with response inhibition is the frontal (or "inhibitory") P3, also generally reported on successful no-go trials. This component is observed at medial-frontal sites in adults, approximately 300-500 msec poststimulus, somewhat later than the P3 observed at parietal sites (e.g., Bruin \& Wijers, 2002; Bokura, Yamaguchi, \& Kobayashi, 2001; Strik, Fallgatter, Brandeis, \& Pascual-Marqui, 1998; Kopp, Mattler, Goertz, \& Rist, 1996). The role of the frontal P3 in response inhibition has been the subject of some discussion. Falkenstein et al. (1999) interpret it as the closing of an inhibition window whose onset is marked by the earlier N2 component. Alternatively, Bokura et al. (2001) suggest that larger frontal P3 amplitudes correspond with lower-probability stimuli, similar to Nieuwenhuis et al.'s (2003) interpretation of the frontal N2.

Because both of these ERPs have been linked with the inhibition of prepotent or impulsive responses, we assumed that they would tap cognitive processes involved in emotion regulation. Is there evidence to suggest a connection with emotion? Indeed, negative emotional evaluations have been found to predict higheramplitude medial-frontal N2s (Tucker, Luu, Desmond, et al., 2003), and a feedback-related negativity, similar to the N2 in topography and morphology, was enhanced by negative evaluations (Luu, Tucker, Derryberry, Reed, \& Poulsen, 2003). Amplitudes of both the feedbackrelated N2 (Tucker, Luu, Frishkoff, et al., 2003) and the frontal P3 (Deldin, Keller, Gergen, \& Miller, 2001) have also been found to correlate with depression. Moreover, Nelson and Nugent (1990) found a frontocentral negativity similar to the N2 in young children, showing larger amplitudes to angry than happy faces, hypothetically indexing attentional processes related to emotional content. The cortical generators associated with medial-frontal ERPs (including the error-related negativity) also suggest links with emotion. Source modeling of these components reveals a key generator in the region of the ACC, a structure thought to integrate cognition and emotion (e.g., Bekker, Kenemans, \& Verbaten, 2005; Luu et al., 2003; Nieuwenhuis et al., 2003; Tucker, Luu, Desmond, et al., 2003; van Veen \& Carter, 2002; Bokura et al., 2001; Fallgatter, Mueller, \& Strik, 1999; Dehaene, Posner, \& Tucker, 1994). This is consistent with the idea that emotion regulation is carried out by the cognitive processes tapped by these components. Similarly, the region of the orbitofrontal cortex (OFC), another area integrating attention and emotion, has been identified as a generator of the N2 and frontal P3 in several studies (Bokura et al., 2001; Pliszka, Liotti, \& Woldorff, 2000). More important, both children and adults show increased fMRI activation in both the ACC and OFC during response inhibition (Casey et al., 1997). Taken together, these findings suggest that ERPs such as the N2 and frontal P3 may tap inhibitory control in response to negative emotional states, as mediated by prefrontal regions including the ACC and possibly the OFC.

Having selected a pair of ERPs that tap response inhibition and having addressed their potential role in emotion regulation, we can now ask what is known about the development of these ERPs. Developmental research with go/no-go tasks has consistently shown a decrease in N2 amplitude (and latency) with age (Johnstone, Pleffer, Barry, Clarke, \& Smith, 2005; Davis et al., 2003; Jonkman, Lansbergen, \& Stauder, 2003). This profile is to be expected because it matches fMRI evidence for decreasing prefrontal activity with age in attentional control tasks. However, inhibitory P3 results are mixed: 6-year-olds showed higher-amplitude P3s than adults on no-go trials as well as more difficult go trials (Davis et al., 2003), but Jonkman et al. (2003) report the absence of a no-go P3 for children, and Johnstone et al. (2005) report smaller anterior P3s for children than adults. These discrepancies are difficult to 
reconcile because all tasks assessed inhibitory control using a go/no-go procedure.

The mixed findings reviewed here suggest the need for systematic developmental research into ERPs tapping inhibitory control. However, a number of design shortcomings should be addressed by this research. First, the tasks utilized for developmental studies are generally boring. Whereas adults are able to attend to boring tasks for long periods of time in the service of long-term rewards (e.g., participant payments), children are notoriously unable to do so. Hence, age differences in the neural correlates of executive processes are probably conflated with age differences in motivational proclivities, endurance, and fatigue effects, and these factors may contribute greatly to discrepancies among studies. Second, developmental studies usually compare one age group with adults (but see Davies, Segalowitz, \& Gavin, 2004, for an exception). Different studies select different age groups for this comparison, each using different tasks. Consequently, their findings are difficult to integrate into a coherent picture of development. In order to map out a developmental profile in a parameter such as inhibitory control, it is necessary to administer similar or commensurate tasks to children of many different ages. Finally, to date, no ERP studies of children have used source analysis techniques, probably due to the high degree of variability in children's waveforms and the consequent "smearing" of the grand-averaged data. This has precluded developmental comparisons of the cortical regions likely to be associated with the cognitive processes tapped by ERP measures-a critical next step for developmental neuroscience (Segalowitz \& Davies, 2004).

Our design attempted to deal with each of these kinds of problems. First, the task itself was designed to be engaging and, in fact, emotionally compelling to children of all ages by highlighting the gain and loss of points that could be cashed in for a valued prize. The speed of stimulus presentation was also adjusted dynamically, in response to participants' performance accuracy, to maintain an error rate of approximately 50\% (see Garavan, Ross, \& Stein, 1999). Thus, there was no chance that the task was too slow for some participants or too fast for others. Second, rather than compare one age group with adults, we looked at children's ERPs across a broad span of development, from 5 to 16 years. This permitted us to map out a reasonably fine-grained profile of developmental change. Commensurability was ensured by the dynamic adjustment procedure, which produced equivalent error rates across the age span. Finally, we reduced the variability in our data by selecting subjects (in each age group) with well-defined ERP components prior to source modeling. This allowed us to compare source models across age and hence to draw tentative conclusions about developmental differences in regions of activation.

Our paradigm integrated a go/no-go task with an emotion-induction procedure. Children were required to click a button each time a letter appeared but to refrain from clicking when the same letter showed up twice in succession. Every 20 trials, an on-screen window displayed the points they had earned for correct responses. In order to insure motivation, the children were shown desirable toys or gift certificates (depending on age) prior to the procedure, and they were reminded several times that they needed to earn a high number of points to receive one of these rewards. The task was divided into three blocks, and points rose for all children during Block A. However, points began to drop precipitously in Block B and ended up back at zero. This block was expected to induce negative emotions such as anxiety, frustration or anger, and distress. Finally, points rose again during Block $\mathrm{C}$ so that a prize could be awarded after all, yet we expected that the negative emotions induced in Block B would carry over into Block C for many children. Approximately one-third of all trials were no-go trials. This was to ensure an adequate number of successful no-go trials for averaging the N2 and frontal P3 despite a limited number of trials per block to minimize fatigue and boredom.

We expected amplitudes and latencies for correct no-go trials to diminish with age for both ERP components, consistent with ERP and fMRI findings of agerelated decreases in activation suggestive of increasing cortical efficiency. We also predicted higher amplitudes generated by the emotion induction in Block $\mathrm{B}$ and possibly lasting through Block $\mathrm{C}$, reflecting increased efforts at response inhibition in the presence of negative emotion. Finally, we expected source models of the stimulus-locked waveform to reveal age differences in cortical generators, with older children showing regions of activation more similar to those reported in adults.

\section{METHODS \\ Participants}

Participants were 58 English-speaking children ages 5-16 (28 boys), with normal or corrected-to-normal vision, and free of any psychiatric diagnoses. This age span was subdivided into six periods of 2 years each (see Table 1 ). Participants were recruited through a local newspaper and paid CDN $\$ 40.00$ plus a toy or gift certificate for their participation. Ethical approval of the project was obtained from the University of Toronto and the Hospital for Sick Children in Toronto.

\section{Procedure}

Children were accompanied to the laboratory by a parent. Following a brief introduction to the testing environment, electrode sensor nets, and recording system, parental consent and child assent were obtained. Children were informed that they would receive a prize 
Table 1. Number of Participants in Each Age Group Partitioned by Sex

\begin{tabular}{lccc}
\hline & \multicolumn{3}{c}{ Sex } \\
\cline { 2 - 3 } Age Group (yrs) & Male & Female & Total \\
\hline $5-6$ & 1 & 2 & 3 \\
$7-8$ & 6 & 4 & 10 \\
$9-10$ & 7 & 4 & 11 \\
$11-12$ & 7 & 7 & 14 \\
$13-14$ & 3 & 7 & 10 \\
$15-16$ & 4 & 6 & 10 \\
Total & 28 & 30 & 58 \\
\hline
\end{tabular}

for playing the computer game and were shown two toy bins. One of the bins contained small, undesirable toys such as small plastic cars, whereas the second bin contained more desirable, age-appropriate toys such as large action figures, arts and crafts sets, large stuffed animals, games, and $\$ 10.00$ gift certificates from a local music store for the older participants. The children were informed that with successful performance (accumulation of points) in the game they would have their choice of rewards, but that less successful performance would limit their choice to the less desirable toy bin. The children were asked to choose a toy (or gift certificate) they would like to earn. The electrode sensor net was then applied and the child was seated in front of a computer monitor, with the distance and alignment to the monitor controlled by use of a chin rest. Children were instructed to make responses in the game by clicking a button on a response pad with the index finger of their dominant hand (writing hand). Children were given a practice block of 30 trails to ensure proficiency with the task, with the opportunity to repeat the practice block if needed. Children and parents were fully debriefed about the purpose of the study after two more longitudinal waves (to be described in a forthcoming report).

\section{Task}

The emotion-induction go/no-go task used in the present study was partly adapted from a task developed by Garavan et al. (1999) and presented using E-Prime software (Psychological Software Tools, Pittsburgh, PA). In standard go/no-go paradigms, participants are required to press a button as fast as possible given a particular category of stimuli (the go condition) and withhold responding given another category of stimuli (the no-go condition). Our participants were instructed to click the button for each letter presented but to avoid clicking when a letter was repeated a second time in succession. Different pairs of similarly shaped letters were used for each block (Block A: x, y; Block B: o, p;
Block C: u, d) to enhance novelty without modifying the level of difficulty and to facilitate guided recall during a self-report scale administered to a subset of participants. The no-go error rate for the task was maintained at $50 \pm 10 \%$ by adjusting the stimulus duration (and thus the intertrial interval) dynamically. Stimulus duration was increased with each error made on a no-go trial and decreased with each correct response (on no-go trials that followed correct go trials only). This was intended to provide the same level of challenge for all participants at all ages and to obtain a sufficient number of correct no-go trials for ERP averaging. Error feedback was provided by a red bar in the middle of the screen following incorrect responses, omitted responses, and late responses.

Children were reminded at the beginning of the task and the outset of each block that a high number of points was needed to win the "big prize" they had chosen. Every 20 trials, their accumulated points were displayed in red in a window on the screen. Points were added for correct no-go responses and deducted for response errors on both go and no-go trials. Three blocks of trials were presented: A, B, and C. Blocks A and $\mathrm{C}$ were structurally identical, each consisting of 200 trials (including 66 no-go trials in pseudorandom sequence). Block B consisted of 150 trials ( 40 no-go trials), to limit the duration of children's distress during the points-loss condition. In Block A, children saw their points steadily increase, usually to over 1000 . However, changes in the point-adjustment algorithm caused them to lose all their points by the end of Block B. With a return to the more generous algorithm, children then regained their points in Block $\mathrm{C}$ to win the desirable prize. The loss of points in Block $\mathrm{B}$ was intended to induce emotions of anxiety, anger, and/or distress at the possible loss of the prize.

\section{Manipulation Check}

This emotion-induction scheme was tested with a subjective rating scale administered in a second ERP session. This session was conducted with 19 children in the 8-12-year-old range, approximately 12 weeks after the initial testing. At the end of the task, children rated each of the three blocks (using the different letter pairs as reminders) on a 10-point Likert scale for five emotions: "upset," "mad," "nervous," "satisfied," and "excited." Cards showing animated emotion faces of different intensities were used to aid recall.

\section{EEG Data Collection and Analysis}

EEG was recorded using a 128-channel Geodesic Sensor Net (Tucker, 1993) and sampled at $250 \mathrm{~Hz}$, using EGI software (EGI, Eugene, OR). Impedances for all EEG channels were kept below $50 \mathrm{k} \Omega$. All channels were referenced to $\mathrm{Cz}$ (channel 129) during recording. Eye 
blink and eye movement artifacts $(70 \mu \mathrm{V}$ threshold), signals exceeding $200 \mu \mathrm{V}$, and fast transits exceeding $100 \mu \mathrm{V}$ were edited out during the averaging. The EEG was then rereferenced against an average reference (Tucker, Liotti, Potts, Russell, \& Posner, 1993; Bertrand, Perrin, \& Pernier, 1985). Data were filtered using an FIR band-pass filter with a low-pass frequency of $30 \mathrm{~Hz}$ and a high-pass frequency of $1 \mathrm{~Hz}$. Stimulus-locked data were segmented into epochs from $400 \mathrm{msec}$ before to $1000 \mathrm{msec}$ after stimulus onset. Trials with response times shorter than $200 \mathrm{msec}$ or longer than $1000 \mathrm{msec}$ were removed, as they were assumed to reflect nondeliberate behavior. Also, correct no-go trials that did not have a correct go trial preceding and following them were removed because they most likely reflected attentional lapses or chronic nonresponding. Final trial count means were calculated by block (22.86 for Block A, 11.64 for Block B, and 25.19 for Block C) and by age (18.23, 18.88, 18.71, 23.27 , and 20.27 from youngest to oldest). Because there were fewer no-go trials in Block B, we reran tests for amplitude differences by block for both the N2 and P3, with trial count as a covariate, and found very little change in the results. A baseline correction factor was calculated over $400 \mathrm{msec}$ preceding the stimulus.

ERP components were scored using the following criteria: The N2 was coded as the largest negative deflection after the N1 with a medial-frontocentral topography and with a latency between 200 and 500 msec poststimulus. The frontal P3 was coded as the largest positive deflection after the N2 with a medial-frontocentral topography, and with a latency between 250 and $1000 \mathrm{msec}$ poststimulus. Scoring was carried out by a coder blind to the ages of the participants. The latency for each component was recorded as the latency to the peak identified in the amplitude analysis.

\section{Source Analysis}

In order to estimate the cortical generators of the stimuluslocked waveform, temporal-spatial dipole source modeling was performed on non-baseline-corrected, grand-averaged data using brain electrical source analysis (BESA; Berg \& Scherg, 1994). Equivalent dipole models were derived using a spherical head model with an isotropic realistic head approximation factor of 20. Dipoles were consecutively fitted along the stimulus-locked waveform averaged over correct no-go trials, starting approximately $50 \mathrm{msec}$ after the stimulus onset. A final solution was considered adequate when the residual variance was less than 10\% (Berg \& Scherg, 1994).

\section{RESULTS}

In order to identify a fine-grained developmental profile in behavioral and neurophysiological analyses, we subdivided the age span into six periods of 2 years each (ages 5-6, 7-8, 9-10, 11-12, 13-14, 15-16). The youngest group was dropped because it contained only three members, but all other groups contained at least 10, as shown in Table 1. Furthermore, because previous research suggests that boys and girls may differ in the timing of neural development, we entered sex as a covariate for all analyses (both behavioral and ERP) that included age as a factor. Sex effects were observed for a few analyses; however, because none were predicted and these effects were not systematic, we do not report them here.

\section{Behavioral Analyses}

Accuracy Results

Because perseverative responding leads to high accuracy on go trials and low accuracy on no-go trials, and chronic nonresponding leads to high accuracy on no-go trials and low accuracy on go trials, we needed an accuracy measure that combined go and no-go accuracy scores in some reasonable fashion. We chose the simplest measure possible: an average of go and no-go accuracy scores, computed by averaging the means for each trial type. Low scores on either go or no-go trials would pull down the mean, yielding a lower overall score. Using this measure, we found a main effect of age, with older children showing higher accuracy than younger children, $F(4,49)=8.67, p<.001$ (see Figure 1). Planned contrasts revealed lower accuracies for the 7-8- and 9-10-year-olds than for all older

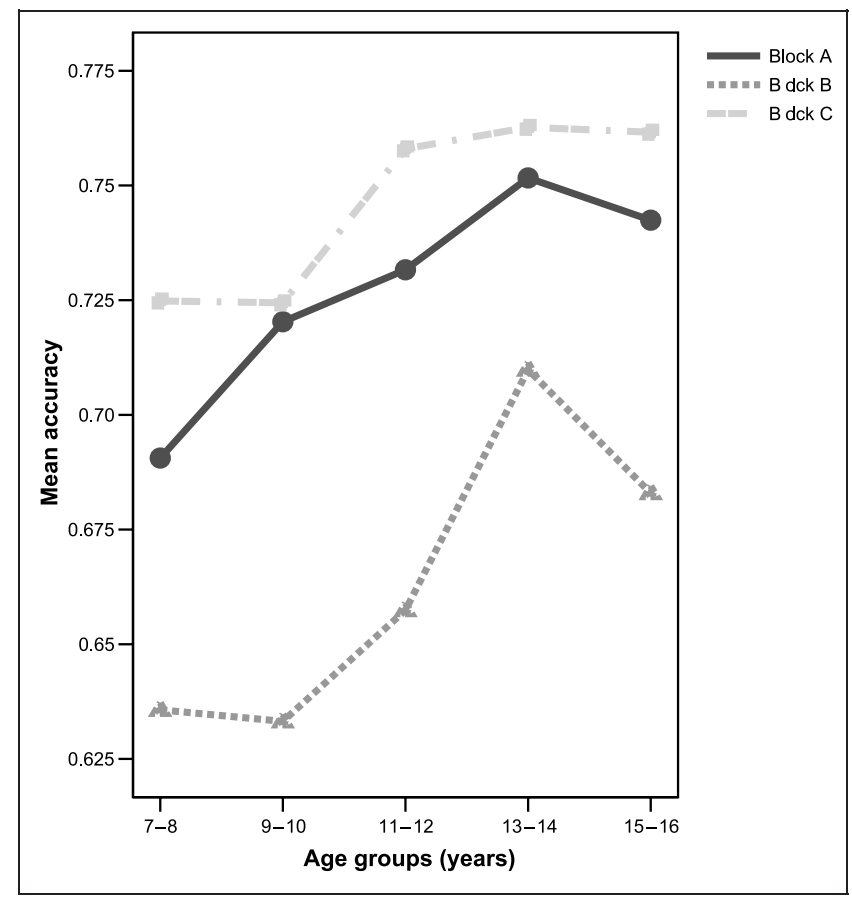

Figure 1. Age differences in accuracy by block. Accuracy was computed by averaging the means for go and no-go trials. 


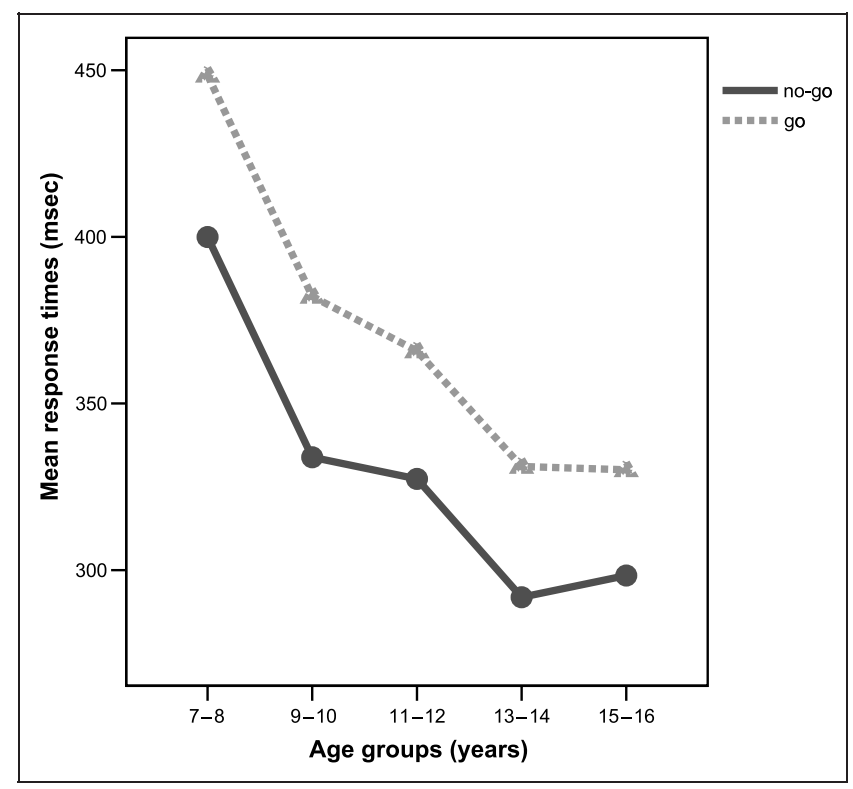

Figure 2. Age differences in response times for go and no-go conditions, collapsing across blocks.

groups ( $p$ values ranging between .03 and $<.001$ ), and lower accuracies for the 11-12- than the 13-14-year-olds $(p=.02)$. As would be expected, a quadratic block effect revealed lowest accuracy scores for Block B (the emotion-induction block), $F(1,49)=33.85, p<$ .001 . Thus, heightened challenge or negative emotion seemed to compromise performance. A quadratic block by age interaction revealed lower accuracy for younger children than older children in Block $\mathrm{B}$, indicating less interference with increasing age, $F(4,49)=4.42, p=$ .004 . This is reflected by the narrowing gap over age between Blocks A and B in Figure 1.

\section{Response Time Results}

Both go and no-go response times were measured from stimulus onset. A main effect of age was found, with older children showing faster response times for both the go condition, $F(4,49)=17.25, p<.001$, and the no-go condition (error trials only), $F(4,49)=11.70$, $p<.001$. Planned contrasts revealed slower response times for the 7-8-year-olds than all other age groups, for both go and no-go conditions $(p<.001)$, slower response times for the 9-10-year-olds than the 13-14and 15-16-year-olds for both conditions ( $p$ values ranging between .03 and .003), and slower response times for the 11-12-year-olds than the 13-14- and 15-16-year-olds for the go condition $(p=.03)$ but only the 13-14-year-olds for the no-go condition $(p=$ $.02)$. A 2 (condition) $\times 3$ (block) $\times 5$ (age group) ANOVA, with repeated measures for condition and block, revealed a main effect of condition, indicating that error (no-go) response times were faster than correct (go) response times across age, $F(1,49)=15.74$, $p<.001$. Thus, error trials were associated with more rapid responding, probably indicating greater impulsivity. Figure 2 reveals the linear decrease in response time with age for both conditions, as well as more rapid response times for the no-go trials overall.

\section{ERP Analyses}

We began the ERP analyses by comparing amplitudes across age at three frontocentral-medial sites (11, 6, and 129, corresponding to $\mathrm{Fz}, \mathrm{FCz}$, and $\mathrm{Cz}$, respectively) and selecting the site that showed the greatest mean age differences for each component. This reduced the overall number of comparisons and highlighted the developmental differences that were the focus of this study. Based on this comparison, frontal P3 analyses were conducted at $\mathrm{Fz}$ and $\mathrm{N} 2$ analyses were conducted at FCz. Figure 3 presents the grand-averaged waveforms of the N2 and frontal P3 components (at their respective electrode sites). As mentioned earlier, grand-averaged waveforms can be smeared due to high variability across individual subjects' waveforms, and this is evident for the children in our sample. Furthermore, the amount of

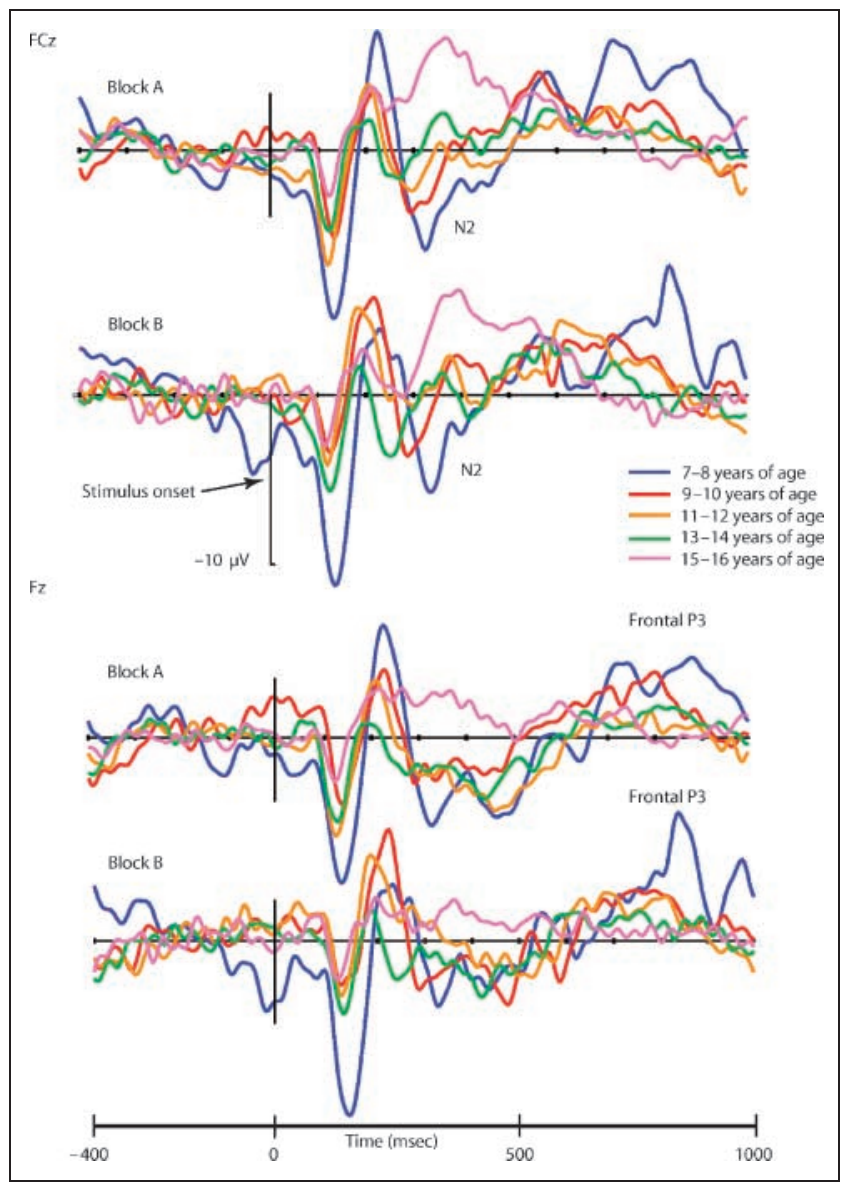

Figure 3. Age and block differences in stimulus-locked, grand-averaged waveforms (for no-go trials) for the N2, at site FCz (top), and the frontal P3, at site Fz (bottom). 


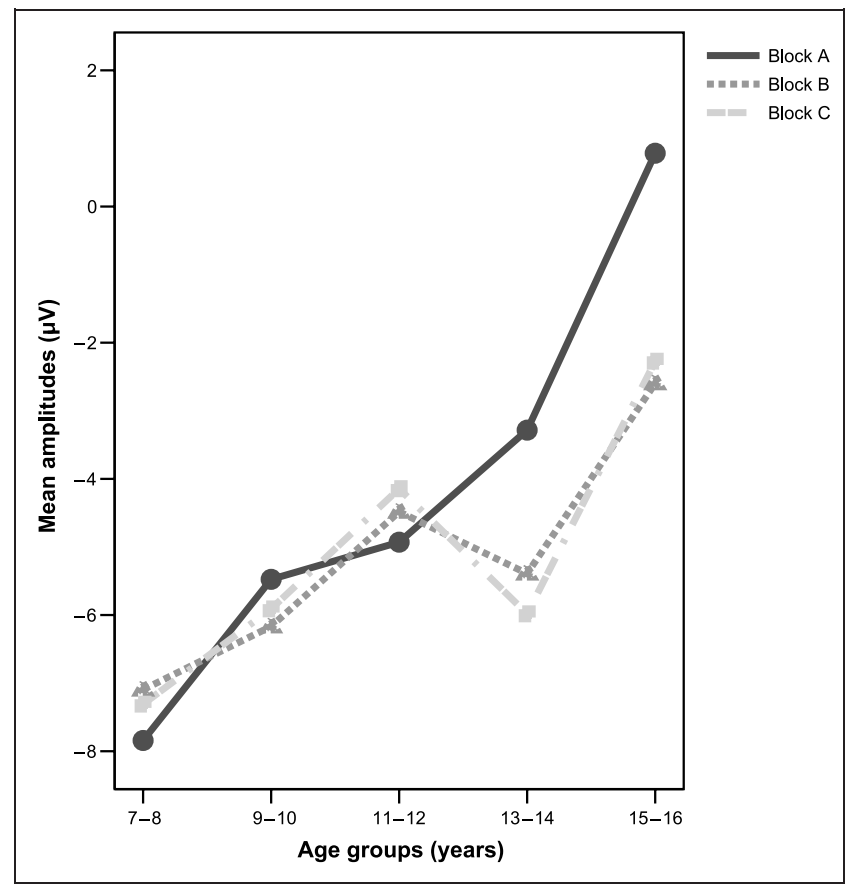

Figure 4. Age differences in N2 amplitudes (at site FCz) by block.

variability can differ greatly from one component to another, as reflected by standard deviations in latency. The mean SDs for the latencies of the N2 and frontal P3, collapsing across blocks and ages, were 45.56 and 183.33 msec, respectively. Thus, latencies were relatively homogeneous for the N2 but heterogeneous for the P3, as reflected in Figure 3.

Figure 3 (top) reveals distinct and sequential age differences in amplitudes and latencies for the N2, especially for Block A. Age differences are evident but much less distinct for the P3, apparently due to high variability in latencies. Because the difference between baseline activation (Block A) and activation during the emotion induction (Block B) is of primary interest here, waveforms for Block $\mathrm{C}$ are not presented. The following statistical analyses examine age and block differences in detail. For each ERP component, we first present age and block results for the no-go trials only, in order to test the hypothesized effects of age and emotion induction on inhibitory control. We then test for differences between go and no-go trials, to better characterize these findings.

\section{Frontal N2 Results}

All N2 results are based on the correct no-go ERP unless otherwise specified. A 5 (age group) $\times 3$ (block) repeated-measures ANOVA (with gender as a covariate) revealed a main effect of age for N2 amplitudes, with smaller amplitudes for older children, as predicted, $F(4,48)=2.66, p=.04$. Planned contrasts showed significantly greater amplitudes for the 7-8- and 9- 10-year-olds than the 15-16-year-olds $(p=.003$ and .02 , respectively). Contrasts also revealed smaller values for the 15-16-year-olds than the 11-12 and 13-14-year-olds at the level of a trend $(p=.08)$. As can be seen in Figure 4, the developmental profile for the N2 is strikingly linear, at least for the A block. However, the emotion-induction (B) block can be seen to increase amplitudes for the oldest two age groups. Indeed, contrasts revealed greater amplitudes for Blocks $\mathrm{B}$ and $\mathrm{C}$ than Block A for the 15-16-year-olds $(p=.04)$, and greater values for Block $\mathrm{C}$ than $\mathrm{A}$ for the 13-14-year-olds $(p=.05)$. Thus, older children appeared more sensitive to the emotion induction or its aftermath. An additional age $\times$ block analysis of $\mathrm{N} 2$ latencies (with identical syntax) also revealed the predicted main effect of age, with older children showing faster latencies, $F(4,48)=10.75, p<.001$. Planned contrasts revealed longer latencies for the 7-8-year-olds than all other age groups ( $p$ values ranging between .03 and $<.001$ ), and longer latencies for the $9-10$ and 11-12-year-olds than the 13-14- and 15-16-year-olds $(p<.005)$. Figure 5 indicates a roughly linear decrease in N2 latencies with age.

To determine if the no-go N2 differed from the go $\mathrm{N} 2$ in amplitude or latency, two 2 (condition) $\times 5$ (age group) $\times 3$ (block) ANOVAs were conducted. Consistent with the literature, N2 amplitudes were greater for no-go trials, $F(1,49)=6.25, p=.02$, and latencies were faster for no-go trials as well, $F(1,49)=13.22$, $p=.001$. We also found a linear condition by block interaction for amplitudes, $F(1,49)=5.43, p=.02$.

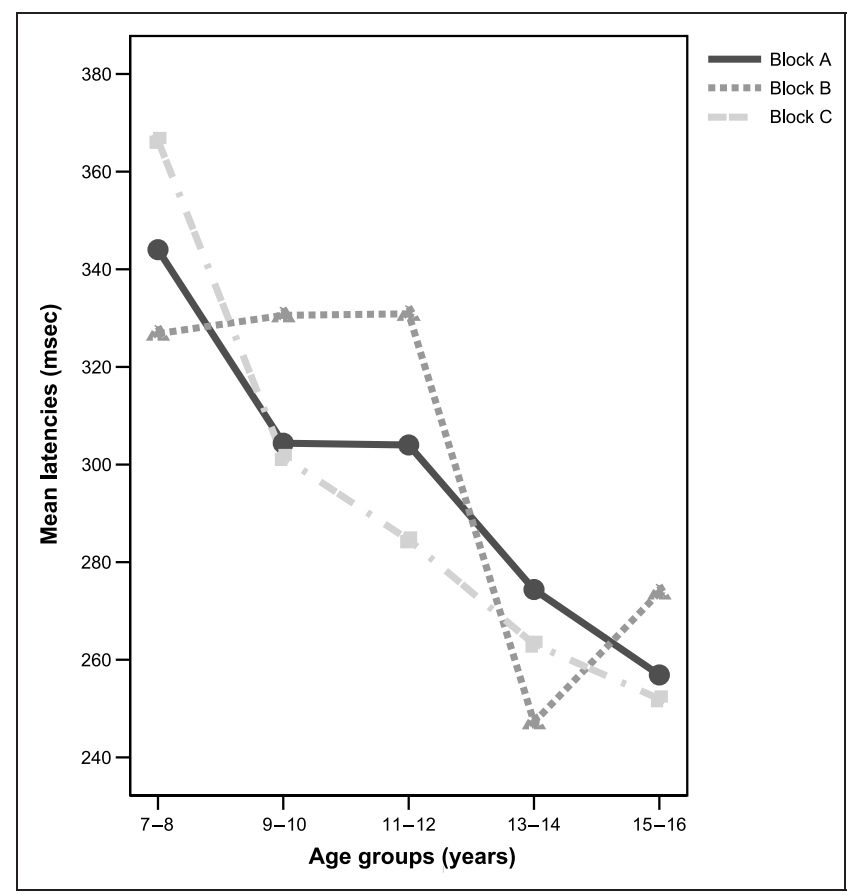

Figure 5. Age differences in $\mathrm{N} 2$ latencies (at $\mathrm{FCz}$ ) by block. 
Bonferroni-corrected contrasts revealed that no-go amplitudes were only greater than go amplitudes for Blocks B $(p=.04)$ and $\mathrm{C}(p=.008)$, suggesting an impact of the emotion induction on processes of inhibitory control. There was no interaction with age. Children of all ages thus showed higher amplitudes and faster latencies when they had to withhold their responses, consistent with the idea that the N2 taps inhibitory control across development.

\section{Frontal P3 Results}

All frontal P3 results are based on the correct no-go ERP unless otherwise specified. A 5 (age group) $\times 3$ (block) repeated-measures ANOVA (with gender as a covariate) revealed a main effect of age for P3 amplitudes, with older children showing smaller values than younger children, as predicted, $F(4,48)=4.75, p=.003$. Planned contrasts showed greater amplitudes for the 7-8-yearolds than the 11-12, 13-14, and 15-16-year-olds ( $p$ values ranging between .009 and $<.001$ ), and greater values for the 9-10-year-olds than the 11-12 and $13-14$-year-olds $(p=.05)$. As can be seen in Figure 6, amplitudes declined relatively steeply at first but then leveled out in the older age range. In addition, as predicted, a quadratic block effect indicated an increase in $\mathrm{P} 3$ amplitudes in Block $\mathrm{B}, F(1,48)=8.59, p=.005$. Planned contrasts revealed greater amplitudes in Block $\mathrm{B}$ than Block A for the 11-12-year-olds $(p=.01)$, and greater values in Block $\mathrm{B}$ than Block $\mathrm{C}$ for the 7-8-, $9-10$, and 11-12-year-olds ( $p$ values range between .04

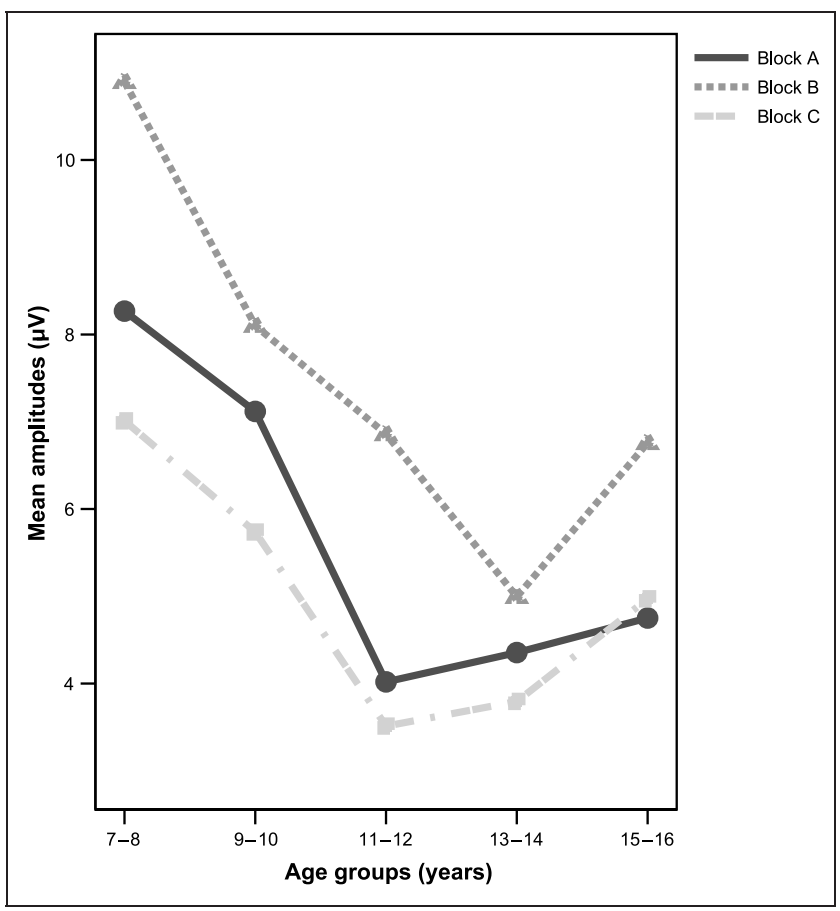

Figure 6. Age differences in frontal P3 amplitudes (at site Fz) by block.

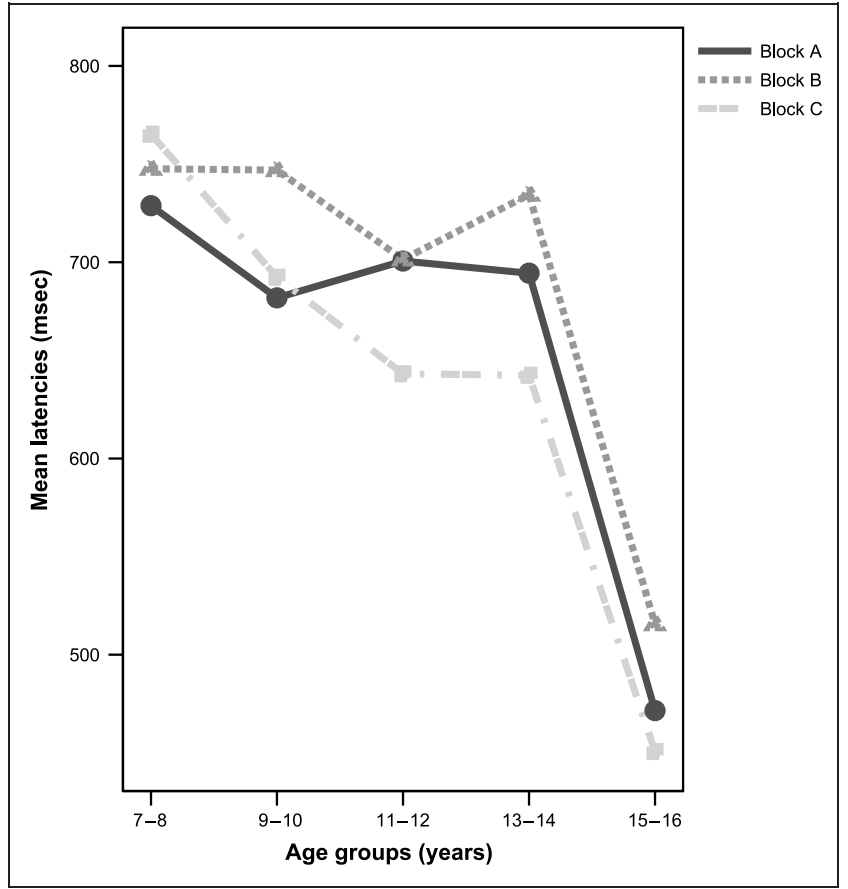

Figure 7. Age differences in frontal $\mathrm{P} 3$ latencies (at site Fz) by block.

and .001). These results indicate that the emotion induction increased cortical activation tapped by the P3 across most of the age range. Frontal P3s not only grew smaller with development but they appeared earlier too, as shown by a main effect of age on latencies, $F(4,48)=5.92, p=.001$, in an additional age $\times$ block ANOVA. Figure 7 indicates a gradual decrease in latencies over the first four age points, followed by a rapid drop for the oldest group. Indeed, contrasts revealed shorter latencies for the 15-16-year-olds than all other age groups $(p<.002)$.

To determine if the no-go P3 differed from the go P3 in amplitude or latency, two 2 (condition) $\times 5$ (age group) $\times 3$ (block) ANOVAs were conducted. Main effects of condition were found for both amplitudes and latencies: No-go amplitudes were greater than go amplitudes, $F(1,49)=116.09, p<.001$, but no-go latencies were longer than go latencies, $F(1,49)=$ $128.69, p<.001$. Thus, frontal P3s were more robust, but later, on trials requiring response inhibition. A quadratic condition by block interaction effect was also revealed, indicating greatest no-go amplitudes, but not go amplitudes, for Block B, $F(1,49)=11.61, p=.001$. Moreover, Bonferroni-corrected contrasts showed greater amplitudes for no-go P3s than go P3s for all three blocks $(p<.001)$, even though Block B was the most augmented. Finally, a linear condition by age interaction effect revealed that no-go P3 amplitudes decreased with age, whereas go $\mathrm{P} 3$ amplitudes did not, $F(4,49)=$ $8.05, p<.001$. This differentiation is clearly shown in Figure 8, which collapses across blocks for ease of viewing. Bonferroni-corrected contrasts revealed greater 


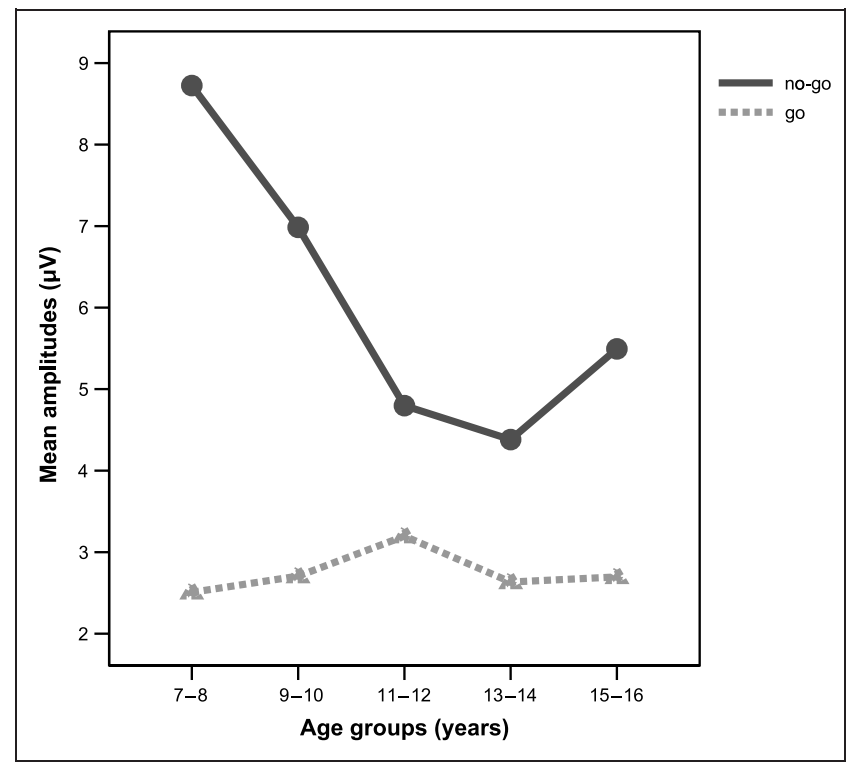

Figure 8. Age differences in frontal P3 amplitudes for go versus no-go conditions (at site Fz), collapsing across blocks.

amplitudes for the no-go condition than the go condition for all age groups ( $p$ values range from .02 to $<.001)$, indicating that no-go amplitudes decreased with age but never to the level of go amplitudes.

\section{Associations with Stimulus Duration}

Our task relied on the dynamic adjustment of stimulus duration to equalize performance difficulty across participants and across age. Although this innovation was useful, it introduced associations between stimulus duration and ERP differences that required further exploration. We first correlated stimulus duration with (the absolute value of) ERP amplitudes. Correlations were positive (for both components) for all three blocks, meaning that children with longer stimulus durations showed greater amplitudes. This was not surprising, because both probably tapped greater effort or poorer competency. Because this association implied shared variance among age, competency, stimulus duration, and ERP amplitudes, we next redid the age analyses with stimulus duration as a covariate. When covariance with stimulus duration was removed, the effect of age on amplitude was reduced. For the P3, the main effect of age remained significant, $F(4,46)=2.70, p<.05$; for the N2, only the contrast for Block A remained significant $(p<.05)$. We also covaried stimulus duration out of the latency analyses and found that the effect of age on latency remained unchanged. Further work will be required to disentangle the effects of age and stimulus duration, both of which may tap some of the same underlying factors.

Mean amplitude was greatest in Block B (for the P3 component), whereas mean stimulus duration was shortest. However, in contrast, amplitude and stimulus duration were positively correlated, as just noted, and their correlation was stronger in Block B $(r=.46, p<$ $.001)$ than in Blocks A and $\mathrm{C}(r \mathrm{~s}=.27$ and .17$)$. This may suggest that longer-than-average stimulus durations in Block $\mathrm{B}$ reflected poorer performance specifically related to the emotion induction. Greater efforts (by some children) to regulate behavior in the presence of negative emotion may have corresponded with higher error rates, thus increasing stimulus duration for those most affected by the emotion induction. Indeed, entering stimulus duration as a covariate reduced the quadratic effect of block on P3 amplitudes below significance. We suggest that this procedure removed much of the effect of the emotion induction itself.

\section{Emotion Scale Results}

A subjective rating scale was used to assess the emotioninduction feature of the task. This scale was administered to a subset of 19 8-12-year-old participants during a second administration of the task. The scale required children to rate the intensity of satisfaction, excitement, nervousness, "upset," and "mad" felt during each block of the task. Analysis of the data revealed a significant quadratic effect for block for all five descriptors. Block B showed the highest mean endorsements for mad, $F(1,18)=15.17, p=.001$, upset, $F(1,18)=$ $13.60, p=.002$, and nervous, $F(1,18)=7.72, p=.01$. Block $B$ also received the lowest mean endorsements for satisfaction, $F(1,18)=46.19, p<.001$, and excitement, $F(1,18)=72.51, p<.001$. The high negative and low positive endorsements for Block B help ascertain that most children felt frustration and/or anxiety when their points were rapidly deducted.

Although we did not plan to use these scores as a reliable index of individual differences in emotion induction, it seemed useful to correlate self-report scores with the amount of change in $\mathrm{P} 3$ amplitude from Block A to $\mathrm{B}$ for this sample (there were no amplitude effects for the N2 in this age range), to determine whether reported negative emotion covaried with the degree of amplitude change. The three negative emotion scales were averaged, and this value was normalized through a log transformation. This composite score was then correlated with the difference score obtained by subtracting Block A amplitudes from Block B amplitudes. With all children included, there was a weak positive correlation between negative emotion scores and amplitude increases, $r=.27, p=n s$. However, when we redid the analysis including only children 10 and older, who were presumably more precise in their self-report, this correlation was strengthened, $r=.54$, one-tailed $p=.05$. These results help support the argument that increasing Block B amplitudes reflected the impact of negative emotion. 


\section{Source Analysis}

Source analysis was conducted to determine if the neural generators underlying children's ERPs varied across age and if the location or activation of these generators changed with the emotion induction. Three steps were undertaken to clean up the variability that can hamper source modeling of child ERP data. First, because N2s showed the least variability in latency, we selected the peak of the N2 component for presentation. Second, to obtain a pool of sufficient size for averaging at several ages, we redivided our sample into three groups of 4 years each. Finally, to clean up amplitude variability, we selected half the children from each of these age groups: those with the highest N2 amplitudes (at site FCz). Though this selection no doubt biased the source models, it did so in a consistent fashion across age, thus allowing meaningful developmental comparisons. Stimulus-locked grand averages were generated for each age group using only the preselected children.

As can be seen in Figure 9 (top), peak N2 activity for the 5-8-year-olds was characterized by a strong centromedial source in the region of the mid- or posterior cingulate cortex for Blocks A and B. As seen in Figure 9 (middle), the 9-12-year-olds showed the same centromedial source, suggesting mid- or posterior cingulate activation, but it appeared weaker for both blocks. A strong right ventral source emerged in Block B as well, suggestive of activation in the OFC or temporal pole. As seen in Figure 9 (bottom), the 13-16-year-olds showed the centromedial source only in Block $\mathrm{B}$, along with a weaker version of the right ventral source shown by the 9-12-year-olds. These children also showed a medialfrontal source in the region of the dorsal ACC, of equivalent strength and appropriate orientation in both Blocks A and B. Inspection of the source models across age suggests a developmental decline in the activation of a mid- or posterior cingulate generator roughly paralleling increased activation of an anterior cingulate generator, indicating a general frontalization of activation with age. Block $\mathrm{B}$ differed from Block A only in the appearance of a right OFC or inferior temporal source for the middle and older age groups. Examination of the topographic plots presented in Figure 9 highlights the decrease in scalp activation with age. Furthermore, an increase in activation from Block A to B is only evident for the oldest group, consistent with the statistical analyses.

\section{DISCUSSION}

Accuracy improved and response time decreased with age, consistent with other studies comparing children with adults (e.g., Jonkman et al., 2003). Moreover, response time was fastest and accuracy was lowest on Block B, the emotion-induction block, at all ages, sug- gesting more impulsive responding when the emotional stakes were raised. Children probably became more "desperate" when they found their points dropping rapidly and they began clicking more indiscriminately as well as more quickly. The increase in errors produced by this response style would have slowed stimulus presentation, given the dynamic adjustment algorithm. However, points would continue to drop (in Block B) regardless of children's efforts, and impulsivity might persist or recur repeatedly as a consequence of ongoing frustration. Interestingly, the tendency toward poorer B-block accuracy diminished with age. Older children may have been better able to inhibit impulsive clicking despite frustration or anxiety, or they could have been less upset, but this too might be considered a consequence of improved regulatory abilities. This trend captures one of the most important, if obvious, gains of growing up: a capability for effortful self-control despite continuing indications of failure. Finally, more rapid responding on no-go trials at all ages indicates that impulsivity may have been partly responsible for errors, as would be expected in a speeded go/no-go task.

N2 amplitudes and latencies decreased with age, as predicted. These findings are consistent with the hypothesis that cortical mechanisms of inhibitory control become more efficient with development (e.g., Casey et al., 2000). Moreover, the reductions in amplitude and latency showed a remarkably linear profile across the five age points, at least for Block A (prior to the emotion induction). This may suggest that developmental improvement in the efficiency of cortical attentional mechanisms continues steadily throughout childhood and adolescence without leveling off until at least late adolescence. However, N2 amplitudes for Blocks B and C branched off this linear trend for children 13-16 years of age. Higher amplitudes following the emotion induction suggest more extensive or more effortful activation of response inhibition mechanisms when anxiety or frustration threatened performance. Yet, our prediction of higher amplitudes beginning with Block B, arguably tapping emotion regulation, was borne out for the older children only. The age by block interaction effect for accuracy, indicating that older children's accuracy deteriorated less in Block $\mathrm{B}$, may reflect the impact of this age-related increase in regulatory efforts.

According to this line of reasoning, older children regulate their behavior in response to anxiety, frustration, or challenge by "amping up" cortical activities that have become more efficient with age (consistent with the suggestion of more effortful processing). However, another overlapping interpretation is that young children were already regulating their behavior at full capacity in Block A. Working to gain points in an unfamiliar game, and wearing an unfamiliar cap on one's head in the presence of strangers, might not be devoid of anxiety, even when one appears to be "winning." Thus, older children may have been less anxious at 
baseline and hence exerting less than maximal effort. It is also conceivable that the gain and loss of points was more meaningful to the older children, making them more sensitive to the change in their success rate in Block B. However, this seems less likely given that younger children expressed the same concerns, felt emotions, and enthusiasm for the prize.

N2 amplitudes were greater for no-go than go trials, as expected, but this difference was specific to Blocks B and $\mathrm{C}$ and thus presumably related to emotion induc-

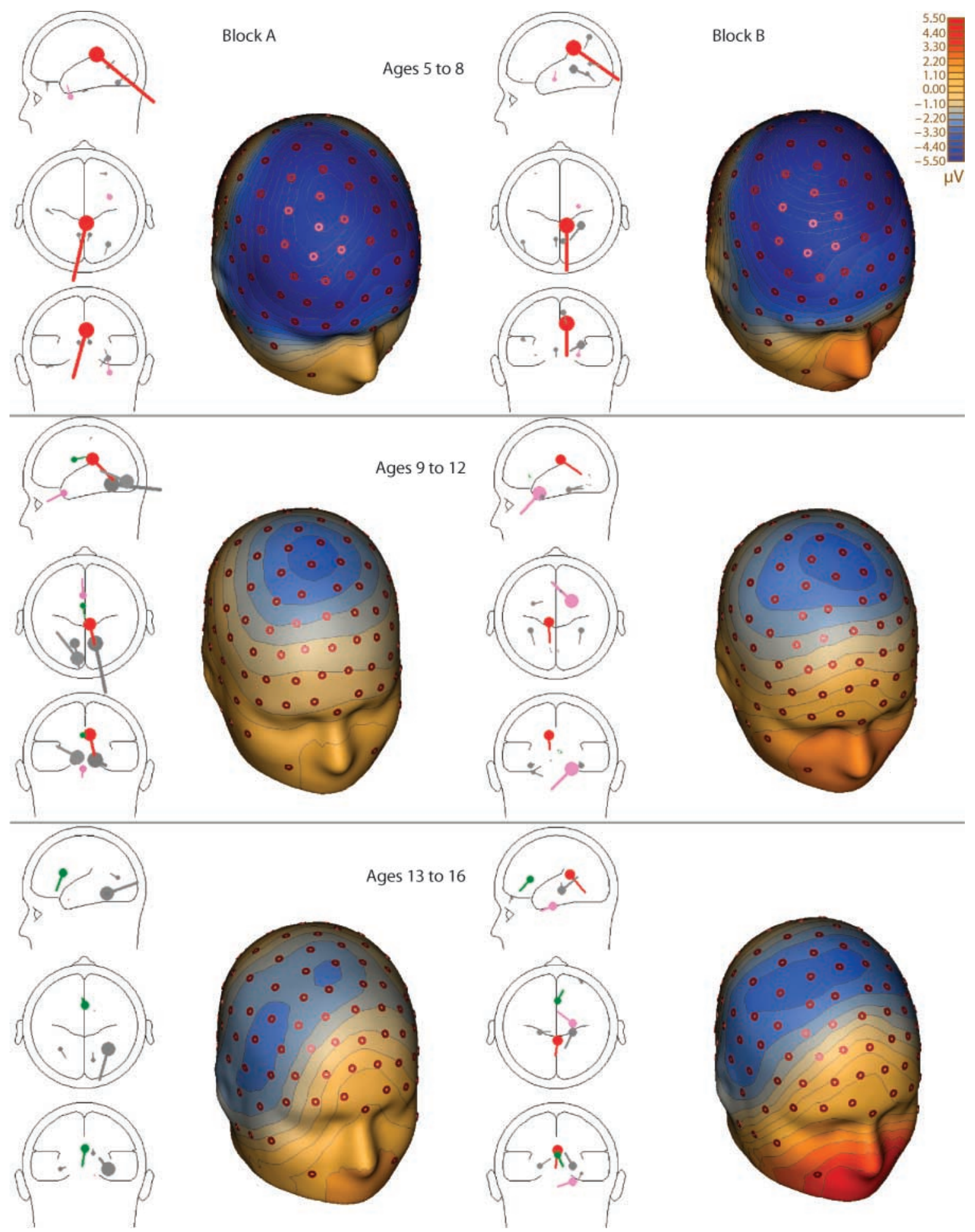

Figure 9. BESA equivalent dipole models for three age groups, based on stimulus-locked, grand-averaged, correct no-go waveforms, shown at peak N2 amplitudes for Blocks A and B. Dipole sizes are proportional to activation strength using the same scale across age groups. 
tion. In studies of adults, both go and no-go trials produce N2s, but most studies report higher amplitudes for no-go trials, consistent with the characterization of the N2 as inhibitory. Studies of children find mixed results. For example, Davis et al. (2003) found no differences between go and no-go amplitudes with 6-year-old children, whereas Johnstone et al. (2005) and Overtoom et al. (1998) report greater amplitudes for no-go trials. It is possible that some emotional highlighting is necessary for children, and possibly adults, to increase their attentional efforts in the service of inhibition as compared with anticipatory evaluation. A task requiring letter discrimination might be particularly boring to children, such that an added motivational component was necessary to maximize the cortical activities underpinning inhibitory control.

Developmental patterns for the frontal P3 were very similar to those for the N2, with both amplitude and latency diminishing over age, consistent with the hypothesis of increasing cortical efficiency. These results contradict Johnstone et al.'s (2005) observation of increasing amplitudes with age but agree with their findings with respect to latency. Given Jonkman et al.'s (2003) finding of no inhibitory P3s in children, one might conclude that task factors and emotional salience make a big difference in the cortical activities tapped by this component. In our data, the relatively steady change in P3 amplitudes and latencies (across five age points) gives us some confidence that the observed developmental decrease is not spurious. We attribute the consistency of our data to the fact that children were highly engaged, motivated, and concerned with their performance at all ages. However, the developmental profile of $\mathrm{P} 3$ amplitudes appeared to level out in the last half of the age range, whereas the developmental profile of P3 latencies dropped most sharply during these years. These results require replication before we attempt to interpret them. Children of all ages showed enhanced $\mathrm{P} 3 \mathrm{~s}$ in the emotion-induction block, consistent with our predictions. Thus, cortical activation mediating inhibitory control again appeared to be greatest in the presence of negative emotions, perhaps reflecting the need for more effortful processing. We cannot explain why P3 amplitudes corresponding with the emotion induction increased at all ages, whereas N2 amplitudes increased only for older children. Perhaps, even for younger children experiencing anxiety in Block A, the frontal P3 was not yet at its ceiling level.

Frontal P3 amplitudes were greater for no-go than go trials. However, unlike the N2, this effect was evident in all blocks. This finding is consistent with studies of the frontal P3 in adults and children (e.g., Johnstone et al., 2005; Fallgatter, Brandeis, \& Strik, 1997) and it points to an inhibitory function tapped by this component. We also observed longer P3 latencies on no-go than go trials. Often, higher-amplitude components are also quicker to appear, but Eimer (1993) reported longer latency
P3s on no-go trials as well. This pattern of results (greater amplitude, longer latency) seems consistent with Falkenstein et al.'s (1999) suggestion that the inhibitory P3 marks the closing of an inhibitory window. Finally, P3 amplitudes decreased with age on no-go trials only. Amplitudes remained low and did not change developmentally for go trials. This finding seems particularly interesting because it integrates the functionality of the frontal P3 with a developmental trend suggesting increased efficiency with age. Whether or not the frontal P3 taps the termination of an inhibitory process, its role in response inhibition appears to require less cortical activation as children mature.

Source modeling suggested a steady developmental decline in the activation of a mid- or posterior cingulate source underlying the N2, roughly paralleled by a developmental increase in the activation of an anterior cingulate source. These results point to a process of frontalization as children mature, consistent with other research (e.g., Bunge et al., 2002; Rubia et al., 2000). An ACC generator for the N2 has frequently been reported for adults (e.g., Nieuwenhuis et al., 2003; Tucker, Luu, Desmond, et al., 2003; van Veen \& Carter, 2002). However, our results suggest that it is not until middle childhood or early adolescence that ACC activity is evident, consistent with Bunge et al.'s (2002) description of immature prefrontal activation in children. An additional source was identified for the two older age groups in the emotion-induction block only, in right inferior frontal or temporal cortex, suggestive of posterior OFC or temporal pole activity. Ventral prefrontal regions have been associated with the processing of negative emotion (Drevets \& Raichle, 1998) or emotion regulation (Davidson, Putnam, \& Larson, 2000), and the right $\mathrm{OFC}$ has been specifically linked to response inhibition (Cunningham, Raye, \& Johnson, 2004). Temporal pole activation has been associated with the induction or reactivation of negative emotions such as anxiety and anger in children as well as in adults (e.g., Nelson et al., 2003; Kimbrell et al., 1999). Thus, in the presence of negative emotion, children in middle childhood and adolescence may have applied ventrally mediated inhibitory controls independent of the deliberate self-monitoring that is generally associated with dorsal prefrontal activities. Finally, it is interesting to note that the oldest two age groups demonstrated more immature source solutions in Block B relative to Block A: the transition to Block $\mathrm{B}$ activated the more posterior midline source for the oldest children and deactivated a small anterior midline source for the 9-12-year-olds. Although merely suggestive, these results imply that response inhibition in the presence of negative emotion recruits cortical activities that are less sophisticated or less developed.

In conclusion, the results of this research indicate a decline in ERP amplitudes and latencies with age for two ERP components tapping inhibitory control. In 
combination with these findings, increased dorsomedial frontal activation with age suggests that cortical activities mediating inhibitory control become both more localized to prefrontal structures and more limited in overall activation. Superimposed on this developmental trend, negative emotion induction produced higher ERP amplitudes specific to response inhibition, with an indication of right ventral activation in middle childhood through adolescence. This pattern of results indicates that inhibitory processes recruited for emotion regulation involve differing cortical regions as children mature.

\section{Acknowledgments}

We gratefully acknowledge the financial support provided by grant No. 1 R21 MH67357-01 from the Developmental Psychopathology and Prevention Research branch of the National Institute of Mental Health (NIMH), as well as support from the Canadian Institutes for Health Research (CIHR) and the Natural Sciences and Engineering Research Council of Canada (NSERC). We are also grateful for support provided (to PDZ) by the Canadian Foundation for Innovation.

Reprint requests should be sent to Marc D. Lewis, Department of Human Development and Applied Psychology, Ontario Institute for Studies in Education, University of Toronto, 252 Bloor Street West, Toronto, Ontario M5S 1V6, Canada, or via e-mail:mlewis@oise.utoronto.ca.

\section{REFERENCES}

Beauregard, M., Levesque, J., \& Bourgouin, P. (2001). Neural correlates of conscious self-regulation of emotion. Journal of Neuroscience, 21, 6993-7000.

Bechara, A., Damasio, H., Tranel, D., \& Damasio, A. R. (1997). Deciding advantageously before knowing the advantageous strategy. Science, 275, 1293-1295.

Bekker, E. M., Kenemans, J. L., \& Verbaten, M. N. (2005). Source analysis of the N2 in a cued Go/NoGo task. Cognitive Brain Research, 22, 221-231.

Berg, P., \& Scherg, M. (1994). A multiple source approach to the correction of eye artifacts. Electroencephalography and Clinical Neurophysiology, 90, 229-241.

Bertrand, O., Perrin, F., \& Pernier, J. (1985). A theoretical justification of the average-reference in topographic evoked potential studies. Electroencephalography and Clinical Neurophysiology, 62, 462-464.

Bishop, S., Duncan, J., Brett, M., \& Lawrence, A. D. (2004). Prefrontal cortical function and anxiety: Controlling attention to threat-related stimuli. Nature Neuroscience, 7, 184-188.

Bokura, H., Yamaguchi, S., \& Kobayashi, S. (2001). Electrophysiological correlates for response inhibition in a Go/NoGo task. Clinical Neurophysiology, 112, 2224-2232.

Botvinick, M. M., Braver, T. S., Barch, D. M., Carter, C. S., \& Cohen, J. D. (2001). Conflict monitoring and cognitive control. Psychological Review, 108, 624-652.

Bruin, K. J., \& Wijers, A. A. (2002). Inhibition, response mode, and stimulus probability: A comparative event-related potential study. Clinical Neurophysiology, 113, 1172-1182.

Bunge, S. A., Dudukovic, N. M., Thomason, M. E., Vaidya, C. J., \& Gabrieli, J. D. E. (2002). Immature frontal lobe contributions to cognitive control in children: Evidence from fMRI. Neuron, 33, 301-311.
Casey, B. J., Giedd, J. N., \& Thomas, K. M. (2000). Structural and functional brain development and its relation to cognitive development. Biological Psychology, 54, 241-257.

Casey, B. J., Trainor, R. J., Orendi, J. L., Schubert, A. B., Nystrom, L. E., Giedd, J. N., Xavier Castellanos, F., Haxby, J. V., Noll, D. C., Cohen, J. D., Forman, S. D., Dahl, R. E., \& Rapoport, J. L. (1997). A developmental functional MRI study of prefrontal activation during performance of a Go-No-go task. Journal of Cognitive Neuroscience, 9, 835-847.

Cunningham, W. A., Raye, C. L., \& Johnson, M. K. (2004). Implicit and explicit evaluation: fMRI correlates of valence, emotional intensity, and control in the processing of attitudes. Journal of Cognitive Neuroscience, 16, $1717-1729$.

Davidson, R. J., \& Irwin, W. (1999). The functional neuroanatomy of emotion and affective style. Trends in Cognitive Sciences, 3, 11-21.

Davidson, R. J., Putnam, K. M., \& Larson, C. L. (2000). Dysfunction in the neural circuitry of emotion regulation-A possible prelude to violence. Science, 289, 591-594.

Davies, P. L., Segalowitz, S. J., \& Gavin, W. J. (2004). Development of response-monitoring ERPs in 7- to 25-year-olds. Developmental Neuropsychology, 25, 355-376.

Davis, E. P., Bruce, J., Snyder, K., \& Nelson, C. (2003). The X-trials: Neural correlates of an inhibitory control task in children and adults. Journal of Cognitive Neuroscience, 13, 432-443.

Dehaene, S., Posner, M. I., \& Tucker, D. M. (1994). Localization of a neural system for error detection and compensation. Psychological Science, 5, 303-305.

Deldin, P. J., Keller, J., Gergen, J. A., \& Miller, G. A. (2001). Cognitive bias and emotion in neuropsychological models of depression. Cognition E Emotion, 15, 787-802.

Derryberry, D., \& Rothbart, M. K. (1997). Reactive and effortful processes in the organization of temperament. Development and Psychopathology, 9, 633-652.

Drevets, W. C., \& Raichle, M. E. (1998). Reciprocal suppression of regional cerebral blood flow during emotional versus higher cognitive processes: Implications for interactions between emotion and cognition. Cognition and Emotion, 12, 353-385.

Durston, S., Thomas, K. M., Yang, Y., Ulug, A. M., Zimmerman, R. D., \& Casey, B. J. (2002). A neural basis for the development of inhibitory control. Developmental Science, 5, F9-F16.

Eimer, M. (1993). Effects of attention and stimulus probability on ERPs in a Go/Nogo task. Biological Psychology, 35, 123-138.

Falkenstein, M., Hoormann, J., \& Hohnsbein, J. (1999). ERP components in Go/Nogo tasks and their relation to inhibition. Acta Psychologica, 101, 267-291.

Fallgatter, A. J., Brandeis, D., \& Strik, W. K. (1997). A robust assessment of the NoGo-anteriorisation of P300 microstates in a cued continuous performance test. Brain Topography, 9, 295-302.

Fallgatter, A. J., Mueller, T. J., \& Strik, W. K. (1999). Age-related changes in the brain electrical correlates of response control. Clinical Neurophysiology, 110, 833-838.

Garavan, H., Ross, T. J., \& Stein, E. A. (1999). Right hemispheric dominance of inhibitory control: An event-related functional MRI study. Proceedings of the National Academy of Sciences, U.S.A., 96, 8301-8306.

Jodo, E., \& Kayama, Y. (1992). Relation of a negative ERP component to response inhibition in a Go/No-go task. Electroencephalography and Clinical Neurophysiology, 82, 477-482. 
Johnstone, S. J., Pleffer, C. B., Barry, R. J., Clarke, A. R., \& Smith, J. L. (2005). Development of inhibitory processing during the Go/NoGo task: A behavioral and event-related potential study of children and adults. Journal of Psychophysiology, 19, 11-23.

Jonkman, L. M., Lansbergen, M., \& Stauder, J. E. A. (2003). Developmental differences in behavioral and event-related brain responses associated with response preparation and inhibition in a go/nogo task. Psychophysiology, 40, $752-761$.

Kimbrell, T. A., George, M. S., Parekh, P. I., Ketter, T. A., Podell, D. M., Danielson, A. L., Repella, J. D., Benson, B. E., Willis, M. W., Herscovitch, P., \& Post, R. M. (1999). Regional brain activity during transient self-induced anxiety and anger in healthy adults. Biological Psychiatry, 46, 454-465.

Kochanska, G., Murray, K., \& Harlan, E. T. (2000). Effortful control in early childhood: Continuity and change, antecedents, and implications for social development. Developmental Psychology, 36, 220-232.

Kopp, B., Mattler, U., Goertz, R., \& Rist, F. (1996). N2, P3 and the lateralized readiness potential in a nogo task involving selective response priming. Electroencephalography $\&$ Clinical Neurophysiology, 99, 19-27.

Lange, K., Williams, L. M., Young, A. W., Bullmore, E. T., Brammer, M. J., Williams, S. C. R., Gray, J. A., \& Phillips, M. L. (2003). Task instructions modulate neural responses to fearful facial expressions. Biological Psychiatry, 53, 226-232.

Luna, B., Thulborn, K., Munoz, D. P., Merriam, E. P., Garver, K. E., Minshew, N. J., Keshavan, M. S., Genovese, C. R., Eddy, W. F., \& Sweeney, J. A. (2000). Maturation of widely-distributed brain function subserves cognitive development. Neuroimage, 13, 786-793.

Luu, P., Tucker, D. M., Derryberry, D., Reed, M., \& Poulsen, C. (2003). Activity in human medial frontal cortex in emotional evaluation and error monitoring. Psychological Science, 14, 47-53.

Nelson, C., \& Nugent, K. M. (1990). Recognition memory and resource allocation as revealed by children's event-related potential responses to happy and angry faces. Developmental Psychology, 26, 171-179.

Nelson, E. E., McClure, E. B., Monk, C. S., Zarahn, E., Leibenluft, E., \& Pine, D. S. (2003). Developmental differences in neuronal engagement during implicit encoding of emotional faces: An event-related fMRI study. Journal of Child Psychology E Psychiatry, 44, 1015-1024.

Nieuwenhuis, S., Yeung, N., Van den Wildenberg, W., \& Ridderinkhof, K. R. (2003). Electrophysiological correlates of anterior cingulate function in a Go/NoGo task: Effects of response conflict and trial-type frequency. Cognitive, Affective \& Behavioral Neuroscience, 3, $17-26$.

Ochsner, K. N., Ray, R. D., Cooper, J. C., Robertson, E. R., Chopra, S., Gabrieli, J. D. E., \& Gross, J. J. (2004). For better or for worse: Neural systems supporting the cognitive down- and up-regulation of negative emotion. Neuroimage, 23, 483-499.

O’Doherty, J., Kringelbach, M. L., Rolls, E. T., Hornak, J., \& Andrews, C. (2001). Abstract reward and punishment representations in the human orbitofrontal cortex. Nature Neuroscience, 4, 95-102.

Overtoom, C. C. E., Verbaten, M. N., Kemner, C., Kenemans, J. L., van Engeland, H., Buitelaar, J. K., Camfferman, G., \& Koelega, H. S. (1998). Associations between event-related potentials and measures of attention and inhibition in the continuous performance task in children with ADHD and normal controls. Journal of the American Academy of Child and Adolescent Psychiatry, 37, 977-985.

Pliszka, S. R., Liotti, M., \& Woldorff, M. G. (2000). Inhibitory control in children with attention-deficit/hyperactivity disorder: Event-related potentials identify the processing component and timing of an impaired right-frontal response-inhibition mechanism. Biological Psychiatry, 48, 238-246.

Posner, M. I., \& Rothbart, M. K. (1998). Attention, self-regulation, and consciousness. Philosophical Transactions of the Royal Society of London, B, 353, 1915-1927.

Rubia, K., Overmeyer, S., Taylor, E., Brammer, M., Williams, S. C. R., \& Simmons, A. (2000). Functional frontalisation with age: Mapping neurodevelopmental trajectories with fMRI. Neuroscience \& Biobehavioral Reviews, 24, 13-19.

Segalowitz, S. J., \& Davies, P. L. (2004). Charting the maturation of the frontal lobe: An electrophysiological strategy. Brain E Cognition, 55, 116-133.

Simpson, J. R., Drevets, W. C., Snyder, A. Z., Gusnard, D. A., \& Raichle, M. E. (2001). Emotion-induced changes in human medial prefrontal cortex: II. During anticipatory anxiety. Proceedings of the National Academy of Sciences, U.S.A., 98, 688-693.

Strik, W. K., Fallgatter, A. J., Brandeis, D., \& Pascual-Marqui, R. D. (1998). Three-dimensional tomography of eventrelated potentials during response inhibition: Evidence for phasic frontal lobe activation. Electroencephalography and Clinical Neurophysiology, 108, 406-413.

Thompson, R. A. (1994). Emotion regulation: A theme in search of definition. The development of emotion regulation: Biological and behavioral considerations. Monographs of the Society for Research in Child Development, 59.

Tucker, D. M. (1993). Spatial sampling of head electrical fields: The geodesic sensor net. Electroencephalography and Clinical Neurophysiology, 87, 154-163.

Tucker, D. M., Liotti, M., Potts, G. F., Russell, G. S., \& Posner, M. I. (1993-1994). Spatiotemporal analysis of brain electrical fields. Human Brain Mapping, 1, 134-152.

Tucker, D. M., Luu, P., Desmond, R. E., Jr., Hartry-Speiser, A., Davey, C., \& Flaisch, T. (2003). Corticolimbic mechanisms in emotional decisions. Emotion, 3, 127-149.

Tucker, D. M., Luu, P., Frishkoff, G., Quiring, J., \& Poulsen, C. (2003). Frontolimbic response to negative feedback in clinical depression. Journal of Abnormal Psychology, 112, 667-678.

van Veen, V., \& Carter, C. S. (2002). The timing of action-monitoring processes in the anterior cingulate cortex. Journal of Cognitive Neuroscience, 14, 593-602.

Wong, P. S., \& Root, J. C. (2003). Dynamic variations in affective priming. Consciousness and Cognition, 12, $147-168$. 